\title{
On complete interpolating sequences and sampling expansions
}

\author{
Kevin Smith
}

\begin{abstract}
Complete interpolating sequences for $L^{2}(-\pi, \pi)$ are considered under the condition that the real parts of the sequence are a subsequence of the scaled integers $x \mathbb{Z}, x>0$. It is found that this condition leads to very specific and restrictive conditions on the existence and structure of complete interpolating sequences for $L^{2}(-\pi, \pi)$. Further general results in the case of bunched sampling of Bernstein functions are also given.
\end{abstract}

\section{Introduction}

A sequence of complex numbers $\left\{z_{m}\right\} \subset \mathbb{C}$, with $z_{m}=x_{m}+i y_{m}$ and $m \in \mathbb{Z}$, is a complete interpolating sequence for $L^{2}(-\pi, \pi)$ if the set $\left\{e^{-i z_{m} \cdot}\right\}$ is complete in $L^{2}(-\pi, \pi)$ and if there exists an $f$ in the Bernstein space $B_{\pi}^{2}$ such that $a_{m}=f\left(z_{m}\right)$ whenever $\left(a_{m}\right) \in \ell^{2}$ (see [4]). Complete interpolating sequences for $L^{2}(-\pi, \pi)$ are of particular significance because a set of complex exponentials $\left\{e^{-i z_{m} \cdot} / \sqrt{2 \pi}\right\}$ is a Riesz basis for $L^{2}(-\pi, \pi)$ if and only if the sequence $\left\{z_{m}\right\}$ is a complete interpolating sequence for $L^{2}(-\pi, \pi)$ (see [4]). Since the Fourier transform is an isometry of $L^{2}$, this yields a series expansion in a Riesz basis of the functions $f$ belonging to the Bernstein space $B_{\pi}^{2}$ (the image of $L^{2}(-\pi, \pi)$ under the Fourier transform) in which the samples $f\left(z_{m}\right)$ arise as the coefficients. A detailed account of this procedure and the theory of Riesz bases can be found in [1], [2] or [4].

Complete interpolating sequences for $L^{2}(-\pi, \pi)$ are also significant in all of the Bernstein spaces $B_{\pi-\epsilon}^{p}$, with $p \leqslant \infty$ and $\epsilon>0$, in the sense that the following holds (a proof based on an extension of Hermite's interpolation formula is given in [1]).

Theorem 1.1. Let $f$ belong to $B_{\pi-\epsilon}^{p}$, with $p \leqslant \infty$ and $\epsilon>0$, and let $\left\{z_{m}\right\}$ be a complete interpolating sequence for $L^{2}(-\pi, \pi)$; then $f$ may be expanded in a series of Lagrange form:

$$
f(z)=\sum_{m} f\left(z_{m}\right) \frac{L(z)}{L^{\prime}\left(z_{m}\right)\left(z-z_{m}\right)},
$$

where

$$
L(z)=\left(z-z_{0}\right) \prod_{n=1}^{\infty}\left(1-\frac{z}{z_{n}}\right)\left(1-\frac{z}{z_{-n}}\right) .
$$

In this paper we consider the existence of complete interpolating sequences for $L^{2}(-\pi, \pi)$ under the restriction that the real parts of the sequence $\left\{z_{m}\right\}, m \in \mathbb{Z}$, are a subsequence of the scaled integers $x \mathbb{Z}=\{x n\}, n \in \mathbb{Z}$, where $x>0$ is a fixed real number. Under this restriction, we find that for $x \geqslant 1 / 2$, the sequence $\left\{z_{m}\right\}$ is a complete interpolating sequence for $L^{2}(-\pi, \pi)$ if and only if $x=(2 n+1) /(4 n+1)$ for $n \in \mathbb{N}_{0}$. It is also found that when $1 / 4<x<1 / 2, x \mathbb{Z}$ contains a unique complete interpolating sequence for $L^{2}(-\pi, \pi)$ if and only if $x=(2 n+1)$ / $(4 n+3)$ for $n \in \mathbb{N}_{0}$. Moreover, it is proved that these complete interpolating subsequences

Received 17 August 2007; revised 20 May 2009.

2000 Mathematics Subject Classification 30E-05, 41A-05, 42A-65, 94-02. 
of $x \mathbb{Z}$ are the bunched sequences $\left\{x_{j k}\right\}=x\{j(4 n+1)+2 k\}$ and $\left\{x_{j k}\right\}=x\{j(4 n+3)+2 k\}$, respectively, where $j \in \mathbb{Z}$ and $k=0, \pm 1, \ldots, \pm n$ in both cases. These results are stated and proved as Theorems 2.2 and 2.3 in the next section.

The sequences in Theorems 2.2 and 2.3 are special cases of a general type known as bunched sequences. In general, the elements of a bunched sequence take the form $z_{j k}=j a+b_{k}$ where $j \in \mathbb{Z}, a \in \mathbb{C},\left\{b_{k}\right\} \subset \mathbb{C}$ and $\left|b_{k}\right|<\infty$. The sequences in Theorems 2.2 and 2.3 also have the property that $b_{k}=-b_{-k}$. It is shown in this paper that the condition $b_{k}=-b_{-k}$ has the consequence that the function $L(z)$ of Theorem 1.1 is an odd periodic function with period $2 a$, and that this fact leads to a particularly simple Lagrange form which can be expressed in terms of the Fourier series of $L(z)$. An interesting property of the Fourier coefficients of $L(z)$ in this case is also noted. As an example in the general case of bunched sampling, we consider the situation where $f$ is a periodic function in $B_{\pi-\pi /(2 n+1)}^{\infty}$ with period $2 n+1$, such that the Lagrange form collapses to a finite sum.

\section{Complete interpolating sequences}

\subsection{Preliminaries}

The following relevant necessary and sufficient condition for the set $\left\{z_{m}\right\}$ to be a complete interpolating sequence for $L^{2}(-\pi, \pi)$ was stated by Kadec [3] (although there was an error in the proof of the general case, with $y_{m} \neq 0$, which was corrected by Young).

TheOREM 2.1. A sequence of complex numbers $\left\{z_{m}\right\}$ is a complete interpolating sequence for $L^{2}(-\pi, \pi)$ if and only if there exist constants $C$ and $D$ such that $\left|y_{m}\right| \leqslant C<\infty$ and

$$
\left|x_{m}-m\right| \leqslant D<1 / 4
$$

for all $m \in \mathbb{Z}$.

Considering a sequence of complex numbers such that $\left\{x_{m}\right\} \subset x \mathbb{Z}$ and $\left|y_{m}\right| \leqslant C<\infty$, the following questions arise.

(i) For which $x$ does there exist a subsequence $\left\{x_{m}\right\} \subset x \mathbb{Z}$ that is a complete interpolating sequence for $L^{2}(-\pi, \pi)$ ?

(ii) When such a subsequence exists, is it unique? (In other words, can there exist a distinct subsequence that is also a complete interpolating sequence for $L^{2}(-\pi, \pi)$ ?)

(iii) Can such subsequences be determined explicitly?

The following corollaries serve as a useful starting point.

Corollary 2.1. If $x>1$, then $x \mathbb{Z}$ does not contain a complete interpolating sequence for $L^{2}(-\pi, \pi)$.

Proof. Since $x>1$, we can find an $n_{0}$ such that $x n_{0}>n_{0}+1 / 4$ and $n_{0}-1<x\left(n_{0}-1\right)<$ $n_{0}-3 / 4$. Thus there does not exist an $m \in \mathbb{N}$ such that $\left|n_{0}-x m\right|<1 / 4$.

Corollary 2.2. If $1 / 2 \leqslant x \leqslant 1$ and $x \mathbb{Z}$ contains a complete interpolating sequence for $L^{2}(-\pi, \pi)$, then the sequence is unique. 
Proof. If $1 / 2 \leqslant x \leqslant 1$, then every open interval of the form $(n-1 / 4, n+1 / 4)$ contains at most one element of $x \mathbb{Z}$.

Corollary 2.3. If $1 / 4 \leqslant x<1 / 2$, then $x \mathbb{Z}$ contains at least one complete interpolating sequence for $L^{2}(-\pi, \pi)$.

Proof. If $1 / 4 \leqslant x<1 / 2$, then every open interval of the form $(n-1 / 4, n+1 / 4)$ contains at least one element of $x \mathbb{Z}$.

Corollary 2.4. If $0<x<1 / 4$, then $x \mathbb{Z}$ contains infinitely many distinct complete interpolating sequence for $L^{2}(-\pi, \pi)$.

Proof. If $0<x<1 / 4$, then every open interval of the form $(n-1 / 4, n+1 / 4)$ contains at least two elements of $x \mathbb{Z}$.

Corollary 2.5. If $x \mathbb{Z}$ contains two distinct complete interpolating sequences for $L^{2}(-\pi, \pi)$, then it contains infinitely many.

Proof. The assumption that $x \mathbb{Z}$ contains two distinct complete interpolating sequences implies that there exists a pair of integers, say $m_{0}$ and $n_{0}$, such that $\left|n_{0}-x m_{0}\right| \leqslant D<1 / 4$ and $\left|n_{0}-x\left(m_{0}+1\right)\right| \leqslant D<1 / 4$. Writing $n_{k}=n_{0}+k p$ and $m_{k}=m_{0}+k q$, we observe that the same equations hold for every $x=p / q$ and every pair $m_{k}$ and $n_{k}$. This establishes the result for rational $x$. For irrational $x$, recall that Kronecker's approximation theorem asserts that the fractional parts of the numbers $\{x n\}, n \in \mathbb{Z}$, are dense in $[0,1]$. This implies the result for irrational $x$.

\subsection{Theorems on complete interpolating subsequences of the scaled integers}

In view of the questions and corollaries arising from Theorem 2.1, we now state and prove the following two theorems.

TheOREM 2.2. If $1 / 2 \leqslant x \leqslant 1$, the sequence $x \mathbb{Z}$ contains a (necessarily unique) complete interpolating sequence for $L^{2}(-\pi, \pi)$ if and only if $x=(2 n+1) /(4 n+1)$ for $n \in \mathbb{N}_{0}$, and the elements of this subsequence are

$$
x_{j k}=x(j(4 n+1)+2 k)
$$

where $j \in \mathbb{Z}$ and $k=0, \pm 1, \ldots, \pm n$.

Proof. Note that the set $(2 n+1) /(4 n+1), n \in \mathbb{N}_{0}$, partitions the closed interval $x \in[1 / 2,1]$ into open subsets $(2 n+1) /(4 n+1)<x<(2 n-1) /(4 n-3), n \in \mathbb{N}$. We shall prove that when $x$ belongs to one of these open subsets, the sequence $x \mathbb{Z}$ does not contain a subsequence that satisfies the conditions of Theorem 2.1. This is achieved by showing that for every such $x$ there exists an open interval $(m-1 / 4, m+1 / 4)$, for some integer $m$, that does not contain an element of $x \mathbb{Z}$. The proof is completed by showing that for $x=(2 n+1) /(4 n+1)$ with $n \in \mathbb{N}_{0}, x \mathbb{Z}$ does in fact contain a (necessarily unique) subsequence that satisfies the conditions of Theorem 2.1 and which is explicitly determined by (4). 
To this end, note that the mid-points of the open intervals $((2 n+1) /(4 n+1),(2 n-1) /$ $(4 n-3))$ are $2\left(4 n^{2}-n-1\right) /((4 n+1)(4 n-3))$, and for each $n \in \mathbb{N}$ the inequality

$$
\frac{2(2 n-1)\left(4 n^{2}-n-1\right)}{(4 n+1)(4 n-3)}<n-\frac{1}{4}<n+\frac{1}{4}<\frac{2(2 n)\left(4 n^{2}-n-1\right)}{(4 n+1)(4 n-3)}
$$

holds. Setting $x=2\left(4 n^{2}-n-1\right) /((4 n+1)(4 n-3))$, this shows that there does not exist an $x_{m} \in x \mathbb{Z}$ that satisfies $\left|x_{m}-n\right|<1 / 4$, so $x \mathbb{Z}$ fails to satisfy (3) in this case. We proceed to rectify this problem in the open interval $(n-1 / 4, n+1 / 4)$ by adjusting $x$ in increasing and decreasing increments. Setting

$$
x=\frac{4 n-1+\epsilon}{4(2 n-1)} \quad \text { or } \quad x=\frac{4 n+1-\epsilon}{8 n}
$$

gives $(2 n-1) x=n-1 / 4+\epsilon$ or $2 n x=n+1 / 4-\epsilon$; thus for sufficiently small $\epsilon$ the problem is rectified. We may re-phrase this statement by stipulating that $x>\alpha_{0}=(4 n-1) /(4(2 n-1))$ or $x<\beta_{0}=(4 n+1) /(8 n)$. Now observe that the inequalities

$$
(6 n-4) \alpha_{0}<3 n-1-\frac{1}{4}<3 n-1+\frac{1}{4}<(6 n-3) \alpha_{0}
$$

and

$$
6 n \beta_{0}<3 n+1-\frac{1}{4}<3 n+1+\frac{1}{4}<(6 n+1) \beta_{0}
$$

hold. Therefore we must choose $x$ such that $x>\alpha_{1}=(12 n-5) /(4(6 n-4))$ or $x<\beta_{1}=$ $(4 n+5) /(4(6 n+1))$. Repeating this procedure for the sequences $\alpha_{1} \mathbb{Z}$ and $\beta_{1} \mathbb{Z}$, we find that we must choose $x>\alpha_{2}=(20 n-9) /(4(10 n-7))$ or $x<\beta_{2}=(20 n+9) /(4(10 n+2))$.

Continuing in this way yields that for any positive integer $k$, we must have

$$
x>\alpha_{k}=\frac{(8 k+4) n-4 k-1}{4((4 k+2) n-3 k-1)} \quad \text { or } \quad x<\beta_{k}=\frac{(8 k+4) n+4 k+1}{4((4 k+2) n+k)} ;
$$

and since

$$
\lim _{k \rightarrow \infty} \alpha_{k}=\frac{2 n-1}{4 n-3} \text { and } \lim _{k \rightarrow \infty} \beta_{k}=\frac{2 n+1}{4 n+1},
$$

we conclude that there does not exist an $x$ in the open intervals $((2 n+1) /(4 n+1)$, $(2 n-1) /(4 n-3)), n \in \mathbb{N}$, such that the sequence $x \mathbb{Z}$ contains a subsequence that satisfies Theorem 2.1.

To prove that for $x=(2 n+1) /(4 n+1), n \in \mathbb{N}_{0}$, the sequence $x \mathbb{Z}$ contains a subsequence as in Theorem 2.1 which is given by (4), let

$$
\begin{aligned}
x_{j k} & =x(j(4 n+1)+2 k) \\
& =j(2 n+1)+\frac{2 k(2 n+1)}{4 n+1},
\end{aligned}
$$

where $j \in \mathbb{Z}$ and $k=0, \pm 1, \ldots, \pm n$, and observe that the inequality

$$
\left|j(2 n+1)+k-x_{j k}\right|=\left|\frac{k}{4 n+1}\right|<\frac{1}{4}
$$

holds. Since $\mathbb{Z}=\{j(2 n+1)+k\}$ when $j \in \mathbb{Z}$ and $k=0, \pm 1, \ldots, \pm n$, the result follows from Corollary 2.2.

TheOREM 2.3. If $1 / 4<x \leqslant 1 / 2$, the sequence $x \mathbb{Z}$ contains a unique complete interpolating sequence for $L^{2}(-\pi, \pi)$ if and only if $x=(2 n+1) /(4 n+3)$ for $n \in \mathbb{N}_{0}$, and the elements of this subsequence are

$$
x_{j k}=x(j(4 n+3)+2 k)
$$

where $j \in \mathbb{Z}$ and $k=0, \pm 1, \ldots, \pm n$. 
Proof. Note that the set $\{1 / 4,(2 n+1) /(4 n+3)\}, n \in \mathbb{N}_{0}$, partitions the closed interval $x \in[1 / 4,1 / 2]$ into open subsets $\{1 / 4<x<1 / 3,(2 n+1) /(4 n+3)<x<(2 n+3) /(4 n+7)\}$, $n \in \mathbb{N}_{0}$. We shall prove that when $x$ belongs to one of these open subsets, the sequence $x \mathbb{Z}$ contains at least two, and hence by Corollary 2.5 infinitely many, distinct subsequences that satisfy the conditions of Theorem 2.1. This is achieved by showing that for every such $x$ there exists an open interval $(m-1 / 4, m+1 / 4)$, for some integer $m$, that contains two elements of $x \mathbb{Z}$. The proof is completed by showing that for $x=(2 n+1) /(4 n+3)$ with $n \in \mathbb{N}_{0}, x \mathbb{Z}$ does in fact contain a unique subsequence that satisfies the conditions of Theorem 2.1 and which is explicitly determined by (13).

To this end, note that the mid-points of the open intervals $((2 n+1) /(4 n+3),(2 n+3) /$ $(4 n+7))$ are $2\left(4 n^{2}+9 n+4\right) /((4 n+3)(4 n+7))$, and for each $n \in \mathbb{N}_{0}$ the inequality

$$
n+\frac{3}{4}<\frac{2(2 n+2)\left(4 n^{2}+9 n+4\right)}{(4 n+3)(4 n+7)}<\frac{2(2 n+3)\left(4 n^{2}+9 n+4\right)}{(4 n+3)(4 n+7)}<n+\frac{5}{4}
$$

holds. Setting $x=2\left(4 n^{2}+9 n+4\right) /((4 n+3)(4 n+7))$, this shows that there exist two elements $x_{m} \in x \mathbb{Z}$ that satisfy $\left|x_{m}-n-1\right|<1 / 4$, so in this case $x \mathbb{Z}$ contains infinitely many distinct subsequences that satisfy (3). We proceed to rectify this problem, so that one element of $x \mathbb{Z}$ remains in the open interval $(n+3 / 4, n+5 / 4)$, by adjusting $x$ in increasing and decreasing increments. Setting

$$
x=\frac{4 n+5}{4(2 n+3)} \quad \text { or } \quad x=\frac{4 n+3}{2(2 n+2)}
$$

gives $(2 n+3) x=n+5 / 4$ or $(2 n+2) x=n+3 / 4$. We may re-phrase this statement by stipulating that $x \geqslant \alpha_{0}=(4 n+5) /(4(2 n+3))$ or $x \leqslant \beta_{0}=(4 n+3) /(4(2 n+2))$. Now observe that the inequalities

$$
3 n+4-\frac{1}{4}<(6 n+9) \alpha_{0}<(6 n+10) \alpha_{0}<3 n+4+\frac{1}{4}
$$

and

$$
3 n+2-\frac{1}{4}<(6 n+5) \beta_{0}<(6 n+6) \beta_{0}<3 n+2+\frac{1}{4}
$$

hold. Therefore we must choose $x$ such that $x \geqslant \alpha_{1}=(12 n+17) /(4(6 n+10))$ or $x \leqslant \beta_{1}=$ $(12 n+7) /(4(6 n+5))$. Repeating this procedure for the sequences $\alpha_{1} \mathbb{Z}$ and $\beta_{1} \mathbb{Z}$, we find that we must choose $x \geqslant \alpha_{2}=(36 n+53) /(4(18 n+31))$ or $x \leqslant \beta_{2}=(36 n+19) /(4(18 n+14))$.

Continuing in this way yields that for any positive integer $k$, we must have

$$
x \geqslant \alpha_{k}=\frac{2 \cdot 3^{k}(2 n+3)-3}{2\left(3^{k}(4 n+7)-3\right)} \quad \text { or } \quad x \leqslant \beta_{k}=\frac{2 \cdot 3^{k}(2 n+1)-3}{2\left(3^{k}(4 n+3)-3\right)} ;
$$

and since

$$
\lim _{k \rightarrow \infty} \alpha_{k}=\frac{2 n+3}{4 n+7} \text { and } \lim _{k \rightarrow \infty} \beta_{k}=\frac{2 n+1}{4 n+3},
$$

we conclude that for every $x$ in the open intervals $((2 n+1) /(4 n+1),(2 n-1) /(4 n-3))$, $n \in \mathbb{N}_{0}$, the sequence $x \mathbb{Z}$ contains infinitely many distinct subsequences that satisfy (3).

We now deal with the open interval $(1 / 4,1 / 3)$, the mid-point of which is $7 / 24$. Setting $x=7 / 24$, we observe that the sequence $x \mathbb{Z}$ contains the elements $7 / 8>3 / 4$ and $7 / 6<5 / 4$, so two elements satisfy $\left|x_{m}-1\right|<1 / 4$. To rectify this so that one element remains in the open interval $(3 / 4,5 / 4)$, we must choose $x \geqslant 5 / 16$ or $x \leqslant 1 / 4$. Thus, by Corollary 2.4 , we are done with the decreasing sequence of increments. For $5 \mathbb{Z} / 16$, note that $45 / 16>3-1 / 4$ and $50 / 16<3+1 / 4$ both belong to $5 \mathbb{Z} / 16$, thus we must choose $x \geqslant 13 / 40$. Continuing in this way, we obtain the sequence $13 / 40,17 / 52, \ldots,(4 k+9) /(12 k+28), \ldots, 1 / 3$. This demonstrates that for $1 / 4<x<1 / 3$, the sequence $x \mathbb{Z}$ contains infinitely many distinct subsequences that satisfy (3). 
To complete the proof, we now show that for $x=(2 n+1) /(4 n+3)$ with $n \in \mathbb{N}_{0}$, the sequence $x \mathbb{Z}$ contains a unique subsequence as in Theorem 2.1 which is given by (13). Since the existence follows from Corollary 2.3, it remains to demonstrate uniqueness. To this end, consider the following finite subsequence of $x \mathbb{Z}$ :

$$
\left\{\frac{k(2 n+1)}{4 n+3}\right\}_{k=-2 n-1}^{2 n}=\left\{\frac{2 k(2 n+1)}{4 n+3}\right\}_{k=-n}^{n} \cup\left\{\frac{(2 k-1)(2 n+1)}{4 n+3}\right\}_{k=-n}^{n} .
$$

Noting that

$$
\left|\frac{(2 k-1)(2 n+1)}{4 n+3}-k\right|=\left|\frac{2 n+k+1}{4 n+3}\right|>\frac{1}{4},
$$

Corollary 2.3 gives that the remaining $2 n+1$ elements consisting of the even multiples $x_{k}=2 k(2 n+1) /(4 n+3)$ must satisfy $\left|x_{k}-k\right|<1 / 4$ for $k=0, \pm 1, \ldots, \pm n$. Letting

$$
\begin{aligned}
x_{j k} & =x(j(4 n+3)+2 k) \\
& =j(2 n+1)+\frac{2 k(2 n+1)}{4 n+3},
\end{aligned}
$$

where $j \in \mathbb{Z}$ and $k=0, \pm 1, \ldots, \pm n$, we identify the unique subsequence of $x \mathbb{Z}$ that satisfies (3) by a one-to-one correspondence.

\section{The Lagrange form in the case of bunched sampling}

The sequences in Theorems 2.2 and 2.3 are special cases of a general type known as bunched sequences. In general, the elements of a bunched sequence take the form $z_{j k}=j a+b_{k}$ where $a \in \mathbb{C},\left\{b_{k}\right\} \subset \mathbb{C}, j \in \mathbb{Z}$ and $\left|b_{k}\right|<\infty$. A particularly simple case (and one which applies to the sequences obtained in Theorems 2.2 and 2.3) occurs when $b_{k}=-b_{-k}$ for $k=0, \pm 1, \ldots, \pm n$.

THEOREM 3.1. Let $\left\{z_{j k}\right\}$ be a bunched complete interpolating sequence for $L^{2}(-\pi, \pi)$ such that $z_{j k}=j a+b_{k}$ where $b_{k}=-b_{-k}$ for $k=0 \pm 1, \ldots, \pm n$ and $j \in \mathbb{Z}$; then

$$
\frac{L(z)}{L^{\prime}\left(z_{m}\right)\left(z-z_{m}\right)}=\frac{a(-1)^{j} \sum_{m=0}^{n} d_{m} \sin (\pi(2 m+1) z / a)}{\pi\left(z-j a-b_{k}\right) \sum_{m=0}^{n} d_{m}(2 m+1) \cos \left(\pi(2 m+1) b_{k} / a\right)}
$$

with

$$
d_{m}=c_{m}-c_{m+1}, \quad c_{m}=(-1)^{m} \sum_{l=m}^{n} 4^{-l}\left(\begin{array}{c}
2 l \\
l-m
\end{array}\right) e_{l},
$$

where $e_{l}$ denotes the lth elementary symmetric polynomial in the $n$ variables $\sin ^{2}\left(\pi b_{1} / a\right), \ldots$, $\sin ^{2}\left(\pi b_{n} / a\right)$.

Proof. Supposing initially that $z$ is real, that $z_{j k}=j a+b_{k}$ satisfies the conditions of Theorem 2.1 and that $b_{k}=-b_{-k}$ with $\left|b_{k}\right|<\infty$, the infinite product

$$
L(z)=\left(z-z_{0}\right) \prod_{m=1}^{\infty}\left(1-\frac{z}{z_{m}}\right)\left(1-\frac{z}{z_{-m}}\right)
$$

reduces to the finite trigonometric product

$$
L(z)=\frac{a}{\pi} \sin \left(\frac{\pi z}{a}\right) \prod_{k=1}^{n} \frac{\sin \left(\pi\left(b_{k}-z\right) / a\right) \sin \left(\pi\left(b_{k}+z\right) / a\right)}{\sin ^{2}\left(\pi b_{k} / a\right)},
$$


a proof of which is obtained by substituting Euler's product formula for the sine in Theorem 1.1. Upon changing variables to $w=2 \pi z / a$, we have

$$
\begin{aligned}
L\left(\frac{a w}{2 \pi}\right) & =\frac{a}{\pi} \sin \left(\frac{w}{2}\right) \prod_{k=1}^{n} \frac{\sin \left(\left(w_{k}-w\right) / 2\right) \sin \left(\left(w_{k}+w\right) / 2\right)}{\sin ^{2}\left(w_{k} / 2\right)} \\
& =\frac{a \sin (w / 2) G(w)}{\pi G(0)},
\end{aligned}
$$

where

$$
\begin{aligned}
G(w) & =\prod_{k=1}^{n} \sin \frac{w_{k}-w}{2} \sin \frac{w_{k}+w}{2} \\
& =\prod_{k=1}^{n} \sin ^{2} \frac{w_{k}}{2}-\sin ^{2} \frac{w}{2}
\end{aligned}
$$

is an even periodic function with period $2 \pi$. The trigonometric product $G(w)$ is the linear factorization of an $n$th order polynomial in $\sin ^{2}(w / 2)$ and, as such, may be written as

$$
G(w)=\sum_{m=0}^{n} e_{m} \sin ^{2 m} \frac{w}{2},
$$

where $e_{m}$ denotes the $m$ th elementary symmetric polynomial in the variables $\sin ^{2}\left(w_{1} / 2\right), \ldots, \sin ^{2}\left(w_{n} / 2\right)$. Since $G(w)$ is an even periodic function with period $2 \pi$, it can be expanded in a Fourier series of the form

$$
G(w)=c_{0}+2 \sum_{m=1}^{n} c_{m} \cos (m w),
$$

where the Fourier coefficients are easily calculated from the polynomial in $\sin ^{2}(w / 2)$ as follows:

$$
\begin{aligned}
c_{m} & =\frac{1}{2 \pi} \int_{0}^{2 \pi} G(w) e^{i m w} d w \\
& =\frac{1}{2 \pi} \sum_{k=0}^{n} e_{k} \int_{0}^{2 \pi} \sin ^{2 k}\left(\frac{w}{2}\right) e^{i m w} d w \\
& =(-1)^{m} \sum_{k=m}^{n} 4^{-k}\left(\begin{array}{c}
2 k \\
k-m
\end{array}\right) e_{k} .
\end{aligned}
$$

Changing variables back to $z=a w / 2 \pi$ gives

$$
\begin{aligned}
L(z) & =\frac{a}{\pi G(0)} \sin \left(\frac{\pi z}{a}\right) G\left(\frac{2 \pi z}{a}\right) \\
& =\frac{a}{\pi} \frac{\sum_{m=0}^{n} d_{m} \sin (\pi(2 m+1) z / a)}{\sum_{m=0}^{n} d_{m}(2 m+1)},
\end{aligned}
$$

where we have written $d_{m}=c_{m}-c_{m+1}$ and $G(0)=c_{0}+2 \sum_{m=1}^{n} c_{m}=\sum_{m=0}^{n}\left(c_{m}-c_{m+1}\right)$ $(2 m+1)$. This is the required Fourier expansion of $L(z)$ for real $z$. Differentiating once with respect to $z$ yields

$$
L^{\prime}(z)=\frac{\sum_{m=0}^{n} d_{m}(2 m+1) \cos (\pi(2 m+1) z / a)}{\sum_{m=0}^{n} d_{m}(2 m+1)} .
$$

Since the sums on the right-hand side are finite sums of entire functions, these representations hold for all $z \in \mathbb{C}$. 


\subsection{An example: periodic functions}

Since the set of all functions with a representation

$$
p(z)=\sum_{m=-n}^{n} c_{m} e^{2 \pi i m z /(2 n+1)}
$$

belongs to $B_{2 \pi n /(2 n+1)}^{\infty}=B_{\pi-\pi /(2 n+1)}^{\infty}$, it follows that Theorem 1.1 applies to any function of this form with $n<\infty$, and we find that

$$
\begin{aligned}
p(z) & =\sum_{j \in \mathbb{Z}} \sum_{k=-n}^{n} p\left((2 n+1)+b_{k}\right) \frac{(-1)^{j} L(z)}{L^{\prime}\left(b_{k}\right)\left(z-j(2 n+1)-b_{k}\right)} \\
& =\sum_{k=-n}^{n} p\left(b_{k}\right) \frac{L(z)}{L^{\prime}\left(b_{k}\right)} \sum_{j \in \mathbb{Z}} \frac{(-1)^{j}}{z-j(2 n+1)-b_{k}} \\
& =\frac{\pi}{2 n+1} \sum_{k=-n}^{n} p\left(b_{k}\right) \frac{L(z)}{L^{\prime}\left(b_{k}\right) \sin \left(\pi\left(z-b_{k}\right) /(2 n+1)\right)} .
\end{aligned}
$$

This kind of expansion is particularly useful in applications when a trigonometric interpolating function is required to interpolate $2 n+1$ non-uniformly spaced points in the vertical strip $-n-1 / 2<\Re(z)<n+1 / 2$. The result asserts that the sampling series coincides with the corresponding Fourier series whenever the points $\left(b_{k}\right)$ satisfy $\left|\Re\left(b_{k}\right)-k\right| \leqslant D<1 / 4$ and $\Im\left(b_{k}\right) \leqslant C<\infty$ for $k=0, \pm 1, \ldots, \pm n$.

A special case of this formula occurs when $b_{k}=k$ (uniform sampling); a little algebra gives

$$
p(z)=\frac{1}{2 n+1} \sum_{k=-n}^{n} p(k) D_{n}\left(\frac{2 \pi(z-k)}{2 n+1}\right),
$$

where $D_{n}(z)=1+2 \sum_{m=1}^{n} \cos (m z)$ is the Dirichlet kernel. This is the series analogue of the integral formula

$$
p(z)=\frac{1}{2 n+1} \int_{n-1 / 2}^{n+1 / 2} p(x) D_{n}\left(\frac{2 \pi(z-x)}{2 n+1}\right) d x,
$$

which is easily obtained on substituting the formula for the Fourier coefficients of $p(z)$ into the corresponding Fourier series.

\subsection{The inverse coefficient mapping}

Theorem 3.1 uses the fact that the Fourier coefficients $c_{m}$ are expressible as linear combinations of the elementary symmetric polynomials $e_{m}$ in the $n$ variables $\sin ^{2}\left(\pi b_{1} / a\right), \ldots, \sin ^{2}\left(\pi b_{n} / a\right)$, which are the coefficients of the polynomial in $\sin ^{2}(\pi z / a)$. The formula which represents this relationship, namely

$$
c_{m}=(-1)^{m} \sum_{l=m}^{n} 4^{-l}\left(\begin{array}{c}
2 l \\
l-m
\end{array}\right) e_{l},
$$

is equivalent to the $(n+1)$-dimensional matrix equation

$$
\left[\begin{array}{c}
c_{0} \\
-c_{1} \\
c_{2} \\
-c_{3} \\
\vdots
\end{array}\right]=\left[\begin{array}{ccccccc}
1 & 2 & 6 & 20 & 70 & 252 & \ldots \\
& 1 & 4 & 15 & 56 & 210 & \ldots \\
& & 1 & 6 & 28 & 120 & \cdots \\
& & & 1 & 8 & 45 & \cdots \\
& & & & 1 & 10 & \cdots \\
& & & & & 1 & \cdots \\
& & & & & & \ddots
\end{array}\right]\left[\begin{array}{c}
e_{0} \\
4^{-1} e_{1} \\
4^{-2} e_{2} \\
4^{-3} e_{3} \\
\vdots
\end{array}\right]
$$

where the $(n+1)$-dimensional unit-diagonal upper triangular matrix is invertible for all $n$. Inverting the matrix, we have 


$$
\left[\begin{array}{c}
e_{0} \\
4^{-1} e_{1} \\
4^{-2} e_{2} \\
4^{-3} e_{3} \\
\vdots
\end{array}\right]=\left[\begin{array}{cccccc}
1 & -2 & 2 & -2 & 2 & \cdots \\
& 1 & -4 & 9 & -16 & \cdots \\
& & 1 & -6 & 20 & \cdots \\
& & & 1 & -8 & \cdots \\
& & & & 1 & \cdots \\
& & & & & \ddots
\end{array}\right]\left[\begin{array}{c}
c_{0} \\
-c_{1} \\
c_{2} \\
-c_{3} \\
\vdots
\end{array}\right]
$$

which is equivalent to the formula

$$
e_{l}=(-4)^{l} \sum_{m=l}^{n}\left[\left(\begin{array}{c}
m+l \\
m-l
\end{array}\right)+\left(\begin{array}{c}
m+l-1 \\
m-l
\end{array}\right)\right] c_{m}
$$

for the inverse mapping.

As an example, we may use this formula to calculate the coefficients $e_{m}$ in the uniform sampling case, that is, when $w_{k}=2 \pi k /(2 n+1)$. In this case we have

$$
\begin{aligned}
G(w) & =(-1)^{n} \prod_{k=1}^{n} \sin \left(\frac{w}{2}+\frac{\pi k}{2 n+1}\right) \sin \left(\frac{w}{2}-\frac{\pi k}{2 n+1}\right) \\
& =\frac{(-1)^{n}}{\sin (w / 2)} \prod_{k=0}^{2 n} \sin \left(\frac{w}{2}+\frac{\pi k}{2 n+1}-\frac{\pi n}{2 n+1}\right) \\
& =\frac{4^{-n} \sin (n+1 / 2) w}{\sin (w / 2)} \\
& =4^{-n} D_{n}(w)
\end{aligned}
$$

where $D_{n}(w)$ is the Dirichlet kernel, which has the Fourier series

$$
D_{n}(w)=1+2 \sum_{m=1}^{n} \cos (m w)
$$

in which $c_{m}=1$. Putting these values into the formula that defines the inverse mapping, we obtain

$$
e_{l}\left(\sin ^{2} \frac{2 \pi}{2 n+1}, \ldots, \sin ^{2} \frac{2 \pi n}{2 n+1}\right)=\frac{(-1)^{l} 4^{l-n}(2 n+1)}{2 l+1}\left(\begin{array}{c}
n+l \\
n-l
\end{array}\right) .
$$

By using elementary trigonometric identities it is also straightforward to obtain

$$
e_{l}\left(\cos ^{2} \frac{2 \pi}{2 n+1}, \ldots, \cos ^{2} \frac{2 \pi n}{2 n+1}\right)=(-1)^{l} 4^{l-n}\left(\begin{array}{c}
n+l \\
n-l
\end{array}\right)
$$

Acknowledgement. The author wishes to thank C. J. Smith, C. S. Smith, J. D. Morrison, D. J. E. Nunn and E. R. Toulson for their support.

\section{References}

1. J. R. Higgins, Sampling theory in Fourier and signal analysis (Oxford University Press, New York, 1996).

2. J. R. Higgins, Completeness and basis properties of sets of special functions (Cambridge University Press, Cambridge, 1977).

3. M. I. KADEC, 'On the exact value of the Paley-Wiener constant', Dokl. Acad. Nauk SSSR 155 (1964) 1253-1254, Soviet Math. Dokl. 5 (1964) 559-561.

4. R. M. Young, An introduction to non-harmonic Fourier series (Academic Press, New York, 1980).

Kevin Smith

159 Thoday Street

Cambridge, CB1 3AT

United Kingdom

k.p.q.smith@googlemail.com 